\title{
¿Educación intercultural para todos? \\ La experiencia escolar de niños indígenas en tres contextos educativos en Ancash
}

\section{Elizabeth Rosales y Alexandra Cussianovich}

Grade

\section{Agradecimientos:}

Agradecemos a Vanessa Rojas y Natalia Streuli por su ayuda en el diseño de este estudio; a Mario Ramírez, por su apoyo durante la preparación de manuales y la recolección de información; a Silvia Velasco y Diana Marchena, por la recolección de información en campo; a Paola Sarmiento, por su apoyo en la fase de análisis; y a Santiago Cueto y Gabriela Guerrero, por supervisar el desarrollo de esta investigación. 


\title{
¿Educación intercultural para todos? \\ La experiencia escolar de niños indígenas \\ en tres contextos educativos
}

\begin{abstract}
Resumen
Esta investigación cualitativa se realizó en el marco del proyecto Niños del Milenio. El objetivo de este artículo es analizar la pertinencia de la experiencia escolar de un grupo de estudiantes indígenas de acuerdo a sus características lingüísticas y culturales. La metodología empleada fue un estudio de casos comparativo en tres escuelas públicas en la región Ancash. Para estudiar la pertinencia de la experiencia escolar de los estudiantes, se emplearon tres ejes de análisis: el uso de la lengua originaria en el aula, la incorporación de conocimientos locales en el currículo y la participación comunitaria en el entorno escolar. Los hallazgos nos llevan a sostener que la experiencia escolar de estos niños no se ajusta a sus necesidades lingüísticas ni culturales, y que la participación de la comunidad en la educación se restringe a la intervención de los padres de familia en cuestiones económicas y materiales de la institución escolar.
\end{abstract}

Palabras clave: educación intercultural, educación bilingüe, diversificación de la educación, participación comunitaria.

\section{Intercultural education for all? The education experience of indigenous children in three educational contexts}

\begin{abstract}
This qualitative research was conducted under the Young Lives project. The aim of this paper is to analyze how pertinent is the schooling experience of indigenous students for their linguistic and cultural characteristics. The methodology was based on a comparative case study in three public schools in the Ancash region. To study the adequacy of the school experience for students, we used three lines of analysis: the use of native language in the classroom, the incorporation of local knowledge in the curriculum, and the community involvement in schools. The findings lead us to argue that the school experience of these children does not meet their language and cultural needs, and community participation in education is restricted to the involvement of parents in economic and material requirements of the school.

Keywords: intercultural education, bilingual education, diversification of education, community participation.
\end{abstract}




\section{Introducción}

a presente investigación forma parte del proyecto Niños del Milenio ${ }^{1}$. Dentro de este marco, en el año 2011, se realizó una Encuesta Escolar con la intención de conocer los procesos educativos de los niños a los que sigue el proyecto. El presente artículo está basado en los hallazgos del subcomponente cualitativo de dicho estudio.

El objetivo central de este estudio es analizar comparativamente la pertinencia de la experiencia escolar de estudiantes indígenas ${ }^{2}$ en tres contextos educativos en la provincia de Huaylas, en Ancash. Asimismo, los objetivos secundarios son comprender en qué medida las interacciones pedagógicas se adaptan a las características lingüísticas y culturales de los estudiantes indígenas, y analizar de qué forma la relación escuela-comunidad fomenta cambios que permitan a la escuela responder a las características de estos niños.

En este sentido, formaron parte de esta investigación tres escuelas públicas que atienden a estudiantes indígenas: una urbana, una rural y una rural EIB. Para estudiar la pertinencia de la experiencia escolar de estos niños, se emplearon tres ejes de análisis: el uso de la lengua originaria en el aula, la incorporación de conocimientos locales en el currículo y la participación comunitaria en el entorno escolar. Estos tres temas estructuran el análisis de resultados que presentamos a continuación, luego de la revisión de literatura y la descripción de la metodología empleada.

\section{Revisión de literatura}

El Perú es un país pluriétnico, pluricultural y plurilingüe. En él, habitan 77 etnias y se hablan 68 lenguas indígenas, además del castellano (Indepa, 2010). La legislación nacional reconoce y pondera esta diversidad, entendiéndola como un recurso para la construcción de una sociedad democrática. En este sentido, una de las medidas orientadas a promover el diálogo y el reconocimiento entre las distintas culturas desde el campo educativo es la Educación Intercultural Bilingüe (EIB).

1. Para obtener mayores detalles sobre el proyecto Niños del Milenio, ver http://www. ninosdelmilenio.org/ y/o http://www.younglives.org.uk/

2. Si bien reconocemos que existe un amplio debate respecto a los criterios empleados para definir el carácter indígena tanto de individuos como de colectivos, para fines de esta investigación, el criterio elegido para determinar la indigeneidad de los participantes ha sido la lengua. 
Si bien aún no existe un concepto único y acabado de EIB (Zavala y Córdova, 2003), es posible afirmar que esta modalidad de educación está basada en el mutuo reconocimiento, respeto y valoración de la diversidad cultural en sociedades donde conviven distintas culturas. La EIB propone un modelo integral, que busca generar canales de intercambio, adquisición y reproducción de saberes y conocimientos entre los miembros de los grupos en contacto, además de impartir una enseñanza bilingüe empleando los idiomas de ambas culturas.

De acuerdo a la propuesta pedagógica EIB del Ministerio de Educación (MED 2012, en consulta), una escuela EIB debe tener como mínimo las siguientes características: docentes formados en EIB que conocen y valoran la cultura de sus estudiantes y manejan su lengua, además del castellano; un currículo que considera los conocimientos, técnicas, historia y valores de la cultura de los estudiantes, así como los conocimientos de otras culturas y de las ciencias; y materiales educativos para las diferentes áreas, tanto en la lengua originaria de los estudiantes como en castellano.

En el año 2003, con la Ley General de Educación28044 se propuso el enfoque intercultural como principio rector en todo el sistema educativo de manera transversal. Con ello, se pretendió universalizar la interculturalidad en todos los niveles, ámbitos y escuelas peruanas, apuntando, entre otras cosas, a la superación de los prejuicios, los estereotipos, la discriminación y el racismo (Ministerio de Educación del Perú, 2004). No obstante, este discurso no ha llegado a transformarse en realidad pues, en la práctica, esta modalidad se ofrece básicamente a algunas poblaciones indígenas de zonas rurales y en el nivel primaria (Zúñiga, 2008).

Sin embargo, con la reciente directiva de la Digeibir para construir un único Registro Nacional de Instituciones Educativas de EIB (RM 0008-2012EI), se ha abierto la posibilidad de incorporar escuelas en zonas urbanas. Tal es el caso de la I.E. "Comunidad Shipiba" de Cantagallo, ubicada en Lima y recientemente reconocida e incorporada en el registro de escuelas EIB del Ministerio de Educación. Si bien el padrón de escuelas EIB aún no ha sido oficializado. Esperamos que la escuela de Cantagallo no sea un caso aislado y que haya una ampliación de esta modalidad educativa a zonas urbanas; y es que para que el diálogo entre culturas se torne efectivo, es importante considerar a las poblaciones indígenas, pero también a la población de la cultura mayoritaria, es decir, castellanohablante (Zavala, Cuenca y Córdova, 2005). Tal como sostiene Carmen López, oficial de educación de Unicef: "poner una lengua en la escuela no nos garantiza una educación intercultural. Tenemos que ir más allá de la lengua en el sentido que debemos articular estos universos culturales distintos a los nuestros" (2012, 12 de junio). 
De lo que se trata, en última instancia, es de dejar de mirar la interculturalidad y el bilingüismo solo como un tema rural y de extender esta modalidad más allá de los espacios "tradicionalmente" indígenas. A modo de ejemplo paradójico, un dato bastante ilustrativo es que Lima constituye la ciudad con mayor población quechua hablante de todo el continente (Zúñiga, 2008). Además de la alta movilidad de las poblaciones indígenas, urge que la EIB sea extendida a contextos urbanos, debido a que esta modalidad educativa apunta a generar un proceso de democratización social y ninguna democracia funciona con la participación de unos pocos.

A pesar de los esfuerzos desplegados tanto desde las instituciones públicas como de organismos de la sociedad civil en torno al tema, vemos que aún no existe un consenso sobre lo que la interculturalidad implica en la práctica. De acuerdo a los lineamientos de política de la EIB emitidos por el Ministerio de Educación, la educación intercultural apunta a promover la inclusión de saberes, conocimientos, valores y prácticas que provienen de diferentes tradiciones culturales, así como el desarrollo de nuevas formas de aprender y enseñar. Sin embargo, es necesario tener en cuenta que la interculturalidad, más que una cuestión metodológica, es una cuestión ideológica. Por ello, debe apuntar a descolonizar el saber y combatir discursos hegemónicos que sostienen la desigualdad, el racismo y la exclusión en la sociedad (Zavala, Cuenca y Córdova, 2005).

En este sentido, la última propuesta pedagógica sobre EIB del Ministerio de Educación (2012) plantea la necesidad de promover una "interculturalidad crítica transformadora", orientada a generar un cambio en la sociedad nacional. Además de valorar y preservar la diversidad, este enfoque pretende ayudar a visibilizar las asimetrías existentes respecto a la población que habla una lengua distinta al castellano o forma parte de una comunidad campesina o indígena.

Ahora bien, en relación con la implementación de la EIB en las escuelas, existen tres aspectos que resultan centrales: el uso de lenguas originarias en el aula, la inclusión de saberes tradicionales en el currículo y la participación de las familias y la comunidad en la educación de los niños. En cuanto al uso de las lenguas vernáculas en los procesos de enseñanza, los estudios realizados en escuelas EIB a inicios del año 2000 reportan que las clases se daban en la lengua local y en castellano de forma alternada (López, 2002; Tapia, 2002). Más recientemente, el informe de la Defensoría del Pueblo sobre EIB a favor de pueblos indígenas (2011) señala que el uso de lenguas originarias está circunscrito a las clases de Comunicación y "aun cuando ya no se prohíbe a los estudiantes hablar en lengua indígena, parece mantenerse la idea de que la escuela es un espacio en el que solo deben hablar en castellano" (Defensoría 
del Pueblo, 2011, p. 521). Para Zúñiga (2008), detrás de la priorización del castellano en el espacio escolar, se encuentra un modelo que busca que los estudiantes se conviertan en monolingües en la lengua de mayor prestigio, el castellano.

La reciente propuesta pedagógica del Ministerio de Educación (2012) busca el desarrollo de la lengua materna de los estudiantes y presta mucha atención a las formas orales para la adquisición de la segunda lengua. Asimismo, aspira a que los alumnos dominen su lengua materna y la segunda lengua en sus formas escritas y orales al finalizar la primaria. Sin embargo, la situación reportada por estudios cualitativos en los últimos años da cuenta del largo camino para lograr estas metas. En cuanto a los usos específicos de la lengua originaria en la escuela, el aymara y el quechua son usados para iniciar la clase en actividades como cantos, preguntas sobre los temas de la clase o recapitular lo visto en la clase anterior (Sanga, 2006; Tapia, 2002). Incluso, este momento era denominado como "la hora del quechua" por una de las docentes estudiadas por Tapia (2002).

Asimismo, Sanga (2006) encontró que las lenguas originarias en los procesos de enseñanza están circunscritas a facilitar la comprensión de los contenidos de la clase e indicaciones de las actividades académicas dichas en castellano. Este mismo autor sostiene que la lengua originaria, también, se emplea para motivar la participación de los estudiantes, así como para controlar la disciplina en el aula. En muy pocas situaciones se fomenta el uso de la lengua originaria en su forma escrita, orientándose más bien su uso hacia las formas orales (Sanga, 2006).

La priorización del castellano en los procesos de enseñanza a niños vernáculo hablantes limita sus posibilidades para desarrollar sus capacidades (Hornberger, 2003). Al comparar los procesos educativos de niños quechua hablantes en una escuela EIB frente a una escuela monolingüe en castellano, Hornberger (2003) resalta la mayor comprensión de los temas tratados en clase que logran los niños en el contexto EIB. Resulta innegable que mientras más usen los niños su lengua materna en la escuela no solo mejorarán su rendimiento académico, sino que también incrementarán su autoestima y tendrán una actitud más positiva hacia la escuela (Ada y Baker 2001, en Zavala y Córdova 2003). En suma, es necesario contextualizar la educación bilingüe y comprender que no existen fundamentos universales para esta. Por ello, resulta esencial analizar las condiciones y características que enmarcan la EIB en nuestro país y en cada escuela en particular.

Asimismo, otro aspecto fundamental para una implementación exitosa de la EIB es la inclusión de saberes tradicionales en la escuela. El modelo propuesto por el Ministerio de Educación (2012) plantea incorporar los saberes 
locales a la escuela para contextualizar y hacer más efectivos los aprendizajes. A través de ello, se busca que los estudiantes se desenvuelvan con pertinencia en su entorno y en otros contextos socioculturales. De este modo, se evita -entre otras cosas- la pérdida de conocimientos locales y el debilitamiento de la identidad sociocultural de los estudiantes, y se fortalece su autoestima. Puntualmente, la propuesta sugiere considerar los aportes de los sabios y expertos de la comunidad en temas como el manejo del medio ambiente, la producción de alimentos, el uso de plantas medicinales, la elaboración de diversos objetos e instrumentos de trabajo; así como saberes relacionados con diferentes expresiones de arte y de espiritualidad.

A este respecto, se ha observado que en muchas escuelas EIB los docentes son conscientes de la importancia de los conocimientos y prácticas locales de las comunidades, y las incorporan en sus prácticas de enseñanza (López, 2002). El reciente informe de la Defensoría del Pueblo (2011) da a conocer que los docentes de escuelas EIB incorporan principalmente saberes referidos al entorno natural, como las flores, animales y plantas. Asimismo, abordan temas que aluden a la historia local y las prácticas sociales y, en menor medida, incorporan las visiones del mundo indígena en el aula.

Si bien esto demuestra un reconocimiento hacia los aspectos culturales, el discurso de los docentes "se estructura a partir de los conceptos e interpretación que se encuentran en los libros de texto o en las guías didácticas con las que orientan su trabajo, y presentan los conocimientos y visiones locales a partir de dicho marco interpretativo" (Defensoría del Pueblo, 2011, p. 518). Es decir, en las escuelas EIB, se presentan elementos locales dentro de la estructura y lógica del conocimiento occidental. Asimismo, se observa que el valor pedagógico de los saberes locales resulta menor al de los contenidos formales que promueve la escuela (Zavala, 2001).

Para que ocurra una incorporación efectiva de los saberes locales en la escuela, que deje de lado el tamiz de la matriz occidental, es necesaria una participación activa y efectiva de las familias y otros miembros de la comunidad en la escuela. Justamente, es este el tercer aspecto central de la EIB. La legislación vigente señala que la sociedad tiene la responsabilidad y el derecho de contribuir al desarrollo de la educación, así como de participar en la definición de las políticas educativas (según la Ley General de Educación 28044). Para complementar, en el Convenio 169 de la Organización Internacional del Trabajo, se establece que los pueblos indígenas deberán ser consultados para el desarrollo de los programas sociales y educativos. A pesar de ello, en la práctica, la participación de los pueblos indígenas y comunidades campesinas o nativas en la definición de las políticas educativas y las propuestas pedagógicas es bastante reducida. Frente a ello, observamos que 
la actual gestión ha empezado a llevar a cabo algunas iniciativas para hacer efectiva la participación de estos grupos en el ámbito educativo, como la Comisión Nacional de Educación Intercultural y Bilingüe - Coneib (, que surge de la Resolución Ministerial0246-2012-ED).

A este respecto, cabe destacar que, según lo sostenido en el Informe de la Defensoría del Pueblo (2011), si bien un $83 \%$ de los padres y madres de familia consultados afirmaron que mantienen una coordinación con la institución educativa de sus hijos, estas coordinaciones están más relacionadas a actividades de índole material y económica (refacción, mantenimiento y construcción de infraestructura escolar, etc.) que a aspectos educativos y formativos de los niños (en los que los padres podrían participar activamente en el aprendizaje de sus hijos). Además, en este informe, se sostiene que la existencia de un grupo de padres vinculados a la institución educativa e involucrados con los aprendizajes de sus niños constituye una oportunidad para desarrollar un trabajo conjunto orientado a fortalecer la EIB.

Afortunadamente, en la actualidad, existen buenos indicios respecto a la valoración de la participación de las familias y la comunidad en la escuela. La propuesta pedagógica de EIB del Ministerio de Educación (2012) sostiene que el enfoque de educación intercultural tiene como aspecto central la incorporación de los saberes locales en los procesos educativos escolares. Ello implica que los padres y madres de familia, además de los sabios y autoridades de la comunidad, tengan un rol fundamental de apoyo al docente en el desarrollo de estos contenidos culturales. Igualmente importante, resulta que sus demandas y expectativas respecto a la educación de sus niños sean recogidas e incorporadas por las autoridades educativas. En esta propuesta, se toma en cuenta que la promoción de la participación de los padres de familia implica también desarrollar metodologías de trabajo que posibiliten un proceso permanente de análisis y reflexión, y permita recoger sus expectativas y demandas.

Si bien la EIB compete directamente a los tres aspectos mencionados anteriormente, se trata también de pensar lo intercultural más allá de la escuela y del mundo indígena, pues la interculturalidad nos involucra a niños, adolescentes y adultos en distintos espacios. En este sentido, resulta imprescindible que exista una correspondencia entre la política educativa para la EIB y el marco político, social y económico que le otorgue sustento para lograr un cambio en la sociedad en su conjunto. Por ello, los fines de la EIB no deben mantenerse aislados de reformas en otros sectores públicos, como los de Economía o Salud, y de cambios políticos acordes con los postulados de la EIB para que deje de ser una propuesta marginal (Zavala y Córdova, 2003). 


\section{Metodología}

Esta investigación se ha guiado por el método de estudio de casos. Se partió de la comparación de tres escuelas y se han incorporado las perspectivas de los propios estudiantes, sus madres y profesores respecto a la experiencia educativa de los niños.

La información fue recogida entre octubre y noviembre de 2011. Se emplearon principalmente cuatro métodos de recolección cualitativos: entrevistas a estudiantes, entrevistas a madres, grupos focales a docentes y observaciones de escuela ${ }^{3}$. Todos estos instrumentos fueron piloteados previamente y contaron con guías que orientaron su uso. Dos trabajadoras de campo fueron entrenadas para la recolección de la información, que duró una semana en cada escuela. Cabe resaltar que una de las trabajadoras de campo dominaba el quechua y se encargó de aplicar las entrevistas a estudiantes y madres que preferían realizar la entrevista en su lengua materna. En total, se entrevistó a 13 estudiantes indígenas entre los 9 y 10 años) y sus madres; también, se realizó 3 grupos focales con docentes ${ }^{4}$ y 12 observaciones de aula ${ }^{5}$ en $3 e r$ y 4 to grado de primaria. Además, se recogió información cuantitativa a través de cuestionarios a docentes ${ }^{6}$, directores y alumnos ${ }^{7}$.

Posteriormente, los audios de las entrevistas y grupos focales fueron transcritos, y se prepararon registros detallados de las observaciones de escuela. Toda esta información fue analizada con ayuda del programa "Atlas ti" durante los meses de enero a marzo de 2012. La data cuantitativa fue procesada y complementó los hallazgos cualitativos.

Como ya se mencionó, para fines de este estudio el criterio elegido para definir la indigeneidad de los participantes ha sido la lengua. Si bien inicialmente nos propusimos trabajar únicamente con niños que tuvieran al quechua como lengua materna, durante el recojo de información decidimos incorporar también a algunos estudiantes castellano-hablantes cuyas madres tenían como lengua materna el quechua. Esto se debió a que consideramos que el contexto familiar y doméstico en el que estos niños se desenvuelven cotidianamente puede ser entendido como indígena en tanto las madres reproducen sus cos-

3. La observación de escuela implicó el registro de la jornada escolar completa y el recreo.

4. Se llevó a cabo un grupo focal por escuela, salvo en la escuela de Huaylas 03, donde se realizó una entrevista a la Directora a partir de los temas del grupo focal.

5. Se realizaron 5 observaciones de aula en las escuelas de Huaylas 02 y 04 . En la escuela de Huaylas 03 solo se realizaron 2 observaciones de aula.

6. Se aplicó un total de 11 encuestas a docentes: 3 en Huaylas 02, 2 en Huaylas 03 y 6 en Huaylas 04.

7. Se realizaron un total de 54 encuestas a estudiantes: 17 en Huaylas 02, 18 en Huaylas 03 y 19 en Huaylas 04. 
tumbres, saberes, creencias y otros elementos culturales particulares. Así, del total de estudiantes participantes, ocho tenían como lengua materna el quechua, mientras cinco eran hijos de madres quechuahablantes.

Para la selección de instituciones educativas, se definieron cuatro grupos de estudio de acuerdo al tipo de escuela a la que asistían los niños del proyecto Niños del Milenio: Privada Urbana Monolingüe, Pública Urbana Monolingüe, Pública Rural Monolingüe y Pública Rural EIB. Posteriormente, se seleccionó intencionalmente una escuela de cada uno de los tres grupos de escuelas públicas en la región Ancash ${ }^{8}$. En cada una de ellas, se buscó tener un equilibrio entre niños y niñas participantes.

A continuación, presentamos una descripción de los tres contextos educativos donde se realizó la investigación. Los datos de las localidades provienen de la Encuesta de Comunidad aplicada como parte del proyecto en el 2009, mientras la información de las escuelas fue proporcionada por sus propios Directores durante la Encuesta Escolar (2011). Cabe mencionar que, si bien las tres escuelas se encuentran en localidades distintas, todas pertenecen a un mismo distrito, ubicado en la provincia de Huaylas, en la región Ancash.

\section{Escuela de Huaylas 02}

La escuela de Huaylas 02 está ubicada en un pequeño poblado rural conformado por 700 habitantes. Gran parte de la población es quechuahablante, mientras el resto es bilingüe en quechua y español. Las principales actividades económicas son la agricultura, la ganadería y la minería de carbón. Esta localidad cuenta con tres instituciones educativas: un Pronoei, una escuela primaria y un colegio secundario.

La escuela de Huaylas 02 es una institución educativa EIB, polidocente completa, pública y mixta de nivel primario y secundario ${ }^{9}$. El número total de alumnos en primaria es de 77.

En general, esta escuela está construida con paredes de adobe, techos de metal corrugado y pisos de concreto. Sin embargo, también cuenta con tres aulas de madera, con techos de metal corrugado y pisos de tierra. Respecto a los servicios básicos, cuenta con luz eléctrica y conexión a internet. El suministro de agua es de un río o un manantial y los baños tienen pozo negro sin tratar.

8. No se identificaron estudiantes indígenas en el tipo de escuelas privada urbana monolingüe.

9. Si bien algunas de las escuelas que formaron parte de este estudio tienen tanto primaria como secundaria, la información contenida en esta investigación está referida solo al nivel primario. 
La escuela tiene algunos espacios para actividades específicas, tales como una biblioteca, una cocina y un comedor para estudiantes, así como una oficina para el director. Asimismo, cuenta con los programas "Una laptop por niño" y Desayunos Escolares del Gobierno Central, y también con un servicio de almuerzos escolares financiado por la Municipalidad Provincial.

\section{Escuela de Huaylas 03}

La escuela de Huaylas 03 se encuentra en una localidad rural mediana que tiene aproximadamente 1500 habitantes. La mayor parte de ellos son quechuahablantes, mientras algunos son bilingües en quechua y español. La población está organizada en una comunidad campesina y se dedica básicamente a actividades agropecuarias. En la comunidad, existe un centro de educación inicial, un Pronoei y una escuela primaria. Hasta 2009, Huaylas 03 contaba con el programa de alfabetización Pronama. Para cursar estudios secundarios, los estudiantes deben caminar 45 minutos hasta la capital distrital o hasta la Escuela de Huaylas 02. La escuela de Huaylas 03 es una escuela pública, mixta, multigrado, de nivel primario que atiende a un total de 78 alumnos. Cuenta con un total de 5 docentes, y uno de ellos atiende a 5 to y 6 to grado. No tiene personal administrativo y la maestra de 3er grado, también, se desempeña como directora de la institución.

Esta escuela tiene paredes de adobe, techos de metal corrugado y pisos de concreto. Cuenta con luz eléctrica, suministro de agua de manantial mediante tuberías y un pozo ciego en los baños. Además, la escuela tiene también una oficina y una cocina. Recibe apoyo del gobierno central a través del programa "Una laptop por niño" y el servicio de Desayunos Escolares.

Asimismo, recibe acompañamiento pedagógico del Programa Estratégico de Logros de Aprendizaje (PELA) del Ministerio de Educación. Sin embargo, los docentes han acordado con el Sutep que nadie puede entrar a las aulas a hacer observaciones, y han negado el acceso a los acompañantes. En esta línea, es importante señalar que esta fue la escuela que mostró mayores resistencias durante el recojo de información en campo. Únicamente, la directora colaboró con el estudio y de manera muy renuente. Por ello, no fue posible llevar a cabo todas las observaciones planificadas ni el grupo focal con docentes en esta institución. 


\section{Escuela de Huaylas 04}

La escuela de Huaylas 04 está ubicada en un centro poblado urbano, que tiene alrededor de 300 habitantes, algunos de los cuales hablan tanto quechua como español, mientras otros solo hablan español. Las principales actividades económicas de la población son la agricultura y la ganadería. La localidad cuenta con servicios educativos en los tres niveles principales: preescolar, primaria y secundaria, y también existe un Pronoei en la zona.

La escuela de Huaylas 04 es una institución educativa pública, mixta, de nivel primario y secundario. La primaria atiende a un total de 227 estudiantes y tiene un total de 11 secciones: 2 secciones por grado, con excepción del 2 do grado que tiene solo una sección. Tiene un total de 11 profesores, uno para cada sección, además de un bibliotecario y 5 personas que se desempeñan como personal administrativo.

Las paredes de la escuela son de ladrillo y cemento, tiene techos de calamina y pisos de concreto. Esta escuela dispone de luz eléctrica y línea telefónica. La provisión de agua es a través de un pozo y los baños cuentan con un pozo ciego. Por otra parte, cuenta con algunos espacios para realizar actividades específicas, como una librería, un laboratorio, una cancha deportiva y un auditorio, así como una oficina para el director. El Municipio Distrital contribuye con personal docente para la institución educativa, que además cuenta con el servicio de Desayunos Escolares.

En líneas generales, observamos que a pesar de que las tres escuelas atienden a niños que tienen al quechua como lengua materna, la única escuela EIB es la de Huaylas 02. De las tres escuelas de la muestra, la escuela de Huaylas 04 es la más grande y está ubicada en un centro poblado urbano, mientras las otras pertenecen al ámbito rural. Las escuelas de ámbitos rurales se encuentran en localidades donde el quechua constituye la lengua preponderante. En correspondencia con ello, en Huaylas 04, se observa una mayor proporción de habitantes que solo habla castellano.

\section{Resultados}

Para observar la pertinencia de la experiencia escolar decidimos enfocarnos en las interacciones pedagógicas. Particularmente, el análisis se centró en las lenguas usadas por docentes y estudiantes en las prácticas de enseñanza- 
aprendizaje, y en la inclusión de saberes locales en los contenidos curriculares. La relación escuela-comunidad ha sido abordada desde el diálogo entre ambas partes, en tanto ello puede incidir en los objetivos educativos que puedan facilitar la adaptación de los estudiantes a la educación formal.

\section{Sobre las lenguas indígenas y la cultura local en el aula}

A partir de las observaciones de aula, podemos señalar que el espacio que ocupa la lengua originaria de los estudiantes en las escuelas visitadas es mínimo. En los tres casos estudiados, el castellano es la principal lengua de comunicación para la enseñanza. El quechua es usado con muy poca frecuencia y solo por algunos de los docentes que observamos.

Esporádicamente, el quechua era usado durante el inicio de las sesiones de clases para responder preguntas del profesor y en situaciones coloquiales o no-académicas dentro del aula. Así pues, se observó a dos niños cantar huaynos en quechua para dar inicio a la clase, así como a otro niño contar una adivinanza en su lengua materna. Asimismo, durante las explicaciones de los docentes, se observó a algunos pocos niños responder a las interrogantes de los docentes en quechua. Se registraron algunos momentos en que el quechua era usado en algunas interacciones coloquiales entre niños, y entre los niños y el docente, para comentar las inasistencias, situaciones familiares o los motivos de riña entre los niños. A continuación, presentaremos algunos ejemplos de estas situaciones. La profesora le pregunta a una niña: “¿qué hace tu mamá?". La niña responde "puchka" (hilar). Luego, la profesora pregunta a los demás niños sobre los quehaceres de sus mamás y les dice que eso son los verbos. (Observación de aula, Huaylas 03). En otro caso, La profesora pregunta a los niños que faltaron el día anterior la razón de su ausencia. Marcela responde en quechua y dice que no asistió a la escuela, porque tuvo que cuidar a su hermana menor, ya que su madre fue a la ciudad. La profesora le pregunta en castellano por qué no trajo a su hermana menor con ella a la escuela. La niña responde nuevamente en quechua y la profesora mantiene una breve conversación en quechua con la niña. La profesora, también, le pregunta en quechua a la niña si no tiene frío, puesto que no viste chompa (Observación de aula, Huaylas 03).

Sin embargo, la mayor parte de las situaciones registradas sobre el uso del quechua en el aula se dieron durante los momentos de exposición o explicación del tema de la clase. En estas situaciones, el docente traducía del castellano al quechua las explicaciones y/o indicaciones que no estaban siendo comprendidas por algunos estudiantes. Este uso de la lengua materna de 
los niños es similar a lo observado por Sanga, quien señala que "la función del aimara en los procesos de enseñanza y aprendizaje es para repetir los contenidos que se enseñan en castellano, el aimara es como un medio de reforzamiento en la comprensión" (2006, p. 80). Ello coincide con lo observado en campo. Durante la clase de Comunicación Integral, luego de la lectura de un texto, la profesora pregunta si la lectura habla de nombres o características. Como ningún alumno contesta, pregunta nuevamente de qué trata la lectura. Un niño responde: "de las bolitas dulces". La profesora se impacienta: "iPero qué dice! ¿Dice que es rico las bolitas? No, a ver piensen... de las cualidades no habla, de los nombres tampoco". Frente al silencio de los niños, ella responde que la lectura nos dice cómo se hacen las bolitas y con qué las hacen. Luego, la profesora pregunta en quechua con qué hacen las bolitas y los niños responden espontáneamente en castellano (Observación de aula, Huaylas 03).

De manera complementaria, en los discursos de los estudiantes y docentes, fue posible notar que existe una plena conciencia de esta función del quechua como lengua facilitadora para la compresión de la instrucción en castellano:

Entrevistadora: [...] Y ella cuando te enseña, ¿en qué lengua te enseña? ¿En castellano o en quechua?

Marcela: Tal vez en quechua, tal vez en castellano.

Entrevistadora: ¿Usa las dos?

Marcela: Sí.

Entrevistadora: Y ¿para qué cosas usa quechua, por ejemplo?

Marcela: Tal vez usa quechua, tal vez no.

Entrevistadora: Pero, ¿por qué a veces habla quechua? ¿Para qué lo utiliza?

Marcela: "Tal vez no entiendes" me dice.

Entrevistadora: Ah, ¿les dice "tal vez no entienden" y ahí les habla en quechua?

Marcela: Sí.

(Entrevista a estudiante, Huaylas 03)

Entrevistadora: [...] ¿También los niños, digamos, participan en castellano en clase o tienden a participar en quechua?

Docente 1: Generalmente, ellos participan en castellano. A veces usted o uno, para que lo puedan entender, tienes que hablarle en quechua, porque no te entienden bien, pero ellos hablan ¿no? No sé si a ustedes les ha pasado... 
Docente 1: ...pero a veces que yo les he explicado algo y los veo perdidos y les hablo quechua, me entienden mejor, pero ellos generalmente te contestan en castellano.

[...]

Docente 3: Se entiende más porque quien te contesta en castellano, pero... Docente 1: Te entiende más en quechua.

Docente 3: En quechua te entiende...

(Grupo focal con docentes, Huaylas 04)

A pesar de que dentro del aula el quechua ocupa un espacio restringido, durante los recreos, los niños conversan y juegan empleando su lengua materna. Los docentes con los que conversamos han percibido el cambio de lengua de sus alumnos en los dos espacios (aula y patio), además de un cambio en su comportamiento. Así, cuando la comunicación tiene lugar al interior del aula en castellano, los niños se muestran poco comunicativos, tímidos y hablan con un volumen de voz muy bajo. En contraste, durante los recreos, los estudiantes interactúan fluidamente en su lengua materna, juegan y hablan en un volumen de voz fuerte.

El comportamiento de los niños indígenas dentro del aula está vinculado a lo que los docentes comentan sobre sus características. Para ellos, la principal característica de los niños quechuahablantes es que son cohibidos, tímidos e introvertidos, lo cual se manifiesta principalmente dentro del contexto del aula, en donde hablan "bajito y con miedo". Llama la atención que los mismos docentes señalen que esta característica desaparece cuando los mismos niños están en el recreo o en las clases de arte o educación física.

Docente: [...] cuando uno programa actividades dentro del aula, hay cierta resistencia, cierta timidez e introversión de los niños a actuar ¿no? Pero lo que me he dado cuenta es que cuando el profesor sale del aula, esos niños que supuestamente son tímidos, son...este... un poco introvertidos, cambian totalmente.

Entrevistadora: ¿Sí?

Docente: De la misma forma he podido observar a la hora de recreo. A ese niño que lo saco a leer, este... un texto, apenas su voz hay que, todos hay que hasta dejar de respirar, creo para que...

Entrevistadora: ...para escucharlo.

Docente: ¡Sí! Para que se pueda escuchar su voz que es un hilito así. A la hora del recreo que bien grita, a la hora de educación física es el que más bulla hace. 
Para los docentes, el espacio de interacción (académico versus no-académico) es lo que estaría determinando la diferencia en el comportamiento de los estudiantes. Sin embargo, se puede deducir de los comentarios de algunos docentes de Huaylas 03 y 04 que la lengua de interacción resulta clave para comprender las diferencias en los comportamientos de los niños.

Entrevistadora: ¿Qué me podría decir de los niños indígenas? ¿Cómo son? Docente: Ah ya. Son cohibidos, ¿no? Tienen el autoestima baja también. [Silencio corto] Pero cohibidos más que todo con la gente extraña o con la gente que le habla castellano, porque si le hablas en su idioma materno se sueltan un poco más de lo acostumbrado, ¿no? Se expresan más, porque será la dificultad de pronunciar las palabras en castellano ¿no? No dicen lo que piensan o lo que quieren. Es más fácil, cuando tú les hablas en quechua entonces agarran confianza y ellos también te hablan en quechua y te hacen las preguntas o te conversan de algún tema, ¿no? Con poco miedo ya, ¿no?

(Grupo focal con docentes, Huaylas 03)

A partir de ello, notamos que la característica de "cohibido o tímido" atribuida al niño indígena tal vez está vinculada a las limitaciones lingüísticas del niño en una escuela que prioriza el castellano como vía principal de comunicación. Como señala Hornberger,

A menudo se dice que los niños quechuas, y en general los educandos indígenas de cualquier parte del mundo, son de naturaleza tímida y reticente; y que ésa es la razón por la que rara vez hablan en clase. De ahí que se nos recomiende no interferir con sus patrones culturales de comportamiento al pedirles que hablen más. A la luz de mis observaciones, sin embargo, pienso que deberíamos preguntarnos si es que esa reticencia no se debe, en gran medida, a que la lengua de la escuela es un idioma totalmente extraño para el niño. (2003, p. 176)

Si bien la escuela prioriza el castellano, se observan pocas prácticas docentes que responden a las características lingüísticas de los estudiantes quechuahablantes. Principalmente, observamos que las modalidades reportadas anteriormente eran empleadas por las profesoras de 3er grado de Huaylas 02 y 03. Es interesante observar que, en los cuestionarios aplicados, los docentes de las tres escuelas declararon que los niños aprenden mejor cuando se les enseña en castellano y quechua, y que consideran que es mejor enseñarles usando ambas lenguas. A pesar de ello, en la práctica solo las dos docentes mencionadas hacen uso efectivo del quechua en el aula. 
En la escuela EIB, nos llamó la atención que solo uno de los dos docentes observados usara el quechua en sus interacciones pedagógicas. Para comprender esta diferencia, analizamos algunos aspectos a nivel individual de los docentes y también aspectos a nivel institucional. A nivel individual, podríamos decir que los dos docentes observados de Huaylas 02 son muy similares en cuanto a su formación inicial, sus años de experiencia en la docencia y el dominio del quechua. Sin embargo, la docente que sí emplea el quechua lleva 16 años más que su colega trabajando en dicha escuela y vive en la misma comunidad donde esta se encuentra ubicada. Ambos elementos podrían indicar que la profesora de 3er grado tiene un conocimiento más cercano de los estudiantes y sus necesidades, y ello podría motivarla a usar el quechua en el aula.

A nivel institucional, también, encontramos aspectos que nos ayudaron a comprender mejor las diferencias observadas en las prácticas pedagógicas de ambos docentes. Durante los grupos focales, al abordar los temas sobre el uso de la lengua materna de los estudiantes y el proceso de diversificación del currículo -ambos aspectos centrales para responder a las características de los estudiantes indígenas y de la propuesta EIB- notamos discrepancias entre los docentes de Huaylas 02. En sus discursos, no se identificó una propuesta pedagógica única para la escuela, si no por el contrario, propuestas individuales.

Entrevistadora: Pero ustedes me mencionaban, por ejemplo, para ustedes, profesora, era bien necesario el quechua en los primeros años ¿no? Primero, segundo, tercero...

Docente 1: Sí, porque el niño mayormente al primer grado llega con su lengua materna, que es el quechua.

Entrevistadora: Uhm.

Docente 1: Y como vienen de diferentes hogares, bueno pues, en el hogar por más que los padres sepan el castellano, no a la perfección, por lo menos pueden defenderse, pero dentro del grupo familiar la comunicación siempre es a través del quechua.

Entrevistadora: Uhm.

Docente 2: En cambio, en mi caso yo lo planteo de otra manera [...]. Yo, en primer grado, lo que suelo hacer es...hacerles una...un bombardeo de palabras que ellos conocen como objetos. Lo que hice en primer grado, objetos: olla, madera, cucharones, plato, así. Bombardearlos a su vez que conocen, pero ponerles su nombre en castellano y ir alimentando su léxico en castellano. Es mi enfoque ¿no?

Entrevistadora: Claro.

Docente 2: Obviamente, es diferente al de la profesora.

Docente 1: Pero es de una manera paulatina profesor. 
Docente 2: No, yo no utilizo quechua, no he utilizado yo quechua, por eso yo le digo.

Docente 1: Bueno, yo sí, por eso le digo de una manera paulatina, porque siempre la comunicación ha sido en quechua en los primeros grados; eso sí le digo.

(Grupo focal con docentes, Huaylas 02)

Como vemos en este extracto del grupo focal de Huaylas 02 , los docentes discuten sobre el uso del quechua al inicio de la primaria. Mientras la Docente 1 considera que es necesario introducir el castellano de una manera "paulatina", el Docente 2 considera que se debe "bombardear" al niño con palabras en castellano desde el inicio de su escolaridad.

Otro de los aspectos donde se observó diferencias entre ambos docentes de la escuela EIB fue en la inclusión de conocimientos de la cultura local en las prácticas de enseñanza. Mientras que en las clases de la profesora de 3er grado se observó el uso de algunas experiencias cotidianas vinculadas al cultivo de la tierra así como los saberes de los niños sobre el valor medicinal de las plantas, en la observación del 4to grado no se registró ninguna situación en la que el docente hiciera referencia a algún conocimiento o práctica local. Las discrepancias observadas nuevamente pueden ser comprendidas a la luz de los comentarios vertidos en los grupos focales; en este caso, en los discursos referidos al proceso de diversificación curricular, uno de los ejes más importantes para el programa de EIB. Como se puede apreciar en la siguiente cita, los profesores señalan que no ha habido una planificación a nivel institucional en cuanto a la incorporación de los conocimientos de la cultura local. Así, el proceso queda en manos de cada docente.

Docente 2: [...] Nosotros hicimos [la diversificación curricular], tuvimos en un grupo de trabajo la profesora y quien le habla en un grupo de trabajo que fue convocado por el Ministerio de Educación.

Docente 2: Y lo hicimos entre siete docentes [...]. Entonces ¿Qué pasa? De los siete, tratamos de ver lo que teníamos en común [...]. Y hicimos una diversificación. Solamente con las cosas comunes.

Entrevistadora: ¿Comunes? Y estos siete docentes ¿pertenecían a qué...a qué zona?

Docente 1: A toda esta zona.

[...]

Docente 2: Acabado el año usted hace una evaluación de su diversificación y tiene que hacer un reajuste.

Docente 1: Reajuste.

Entrevistadora: Ya. 
Docente 2: Para que decir, qué le funcionó, qué no le funcionó y qué le falta.

Entrevistadora: Claro.

Docente 2: Esa parte, hasta ahora no se ha hecho [...]. Seguimos así trabajando con la que se tenía, ahora.

Entrevistadora: ¿No se ha revisado?

Docente 2: Claro, el año pasado lo quisimos hacer, valga la sinceridad, pero el problema es que...

Docente 1: Tiempo.

Docente 2: ...los maestros nos resistimos hay veces por las cuestiones de tiempo.

Docente 1: Sí, ¿no?

Docente 2: Que llegan las vacaciones, que ya nos vamos. Por lo tanto, los maestros, imagino que para este año han cogido esa diversificación y la están aplicando de acuerdo a su punto de vista personal...

Docente 1: Uhm.

Docente 2: ...cuando esto debería de ser a punto de vista de la institución.

(Grupo focal con docentes, Huaylas 02)

Esta situación es un claro ejemplo de lo sostenido por Zúñiga al respecto.

La diversificación, por lo tanto, quedó librada al juicio de los docentes de aula, quienes no han recibido mayor preparación para llevarla adelante y la han interpretado básicamente como la incorporación de algunos elementos de la cultural local (danzas, tradiciones) y el uso de productos (generalmente plantas) de la zona para actividades y confección de juegos y materiales didácticos. En zonas de habla indígena, por ejemplo, la diversificación -bajo la restricción del 30\% para realizarla- no genera una reflexión sobre los saberes indígenas y el lugar que deben ocupar en el currículo. (2008, p.80)

Los docentes de Huaylas 02 reconocen la necesidad de contar con un currículo diversificado y evaluarlo anualmente. Adicionalmente, reclaman la articulación entre primaria y secundaria en este tema específico, tal y como lo sugiere la nueva propuesta de EIB. Empero, los docentes son conscientes de la necesidad de un liderazgo del director para lograrlo.

Docente 1: Eso [evaluación anual de la diversificación] falta hacer creo.

Docente 2: Nos falta hacer.

Entrevistadora: Uhm.

Docente 2: Pero eso todo depende de, de acá de la Dirección pues.

Entrevistadora: Claro. 
Docente 2: Porque los maestros nomás no podemos aventurarnos. ¿Por qué le digo? Porque esto no es un problema de primaria, esto es un problema de la institución.

Entrevistadora: Ya.

Docente 2: Entonces como institución tenemos que hacer indicadores desde primer grado hasta que termine quinto de secundaria.

Docente 1: Quinto de secundaria.

Docente 2: Si no nosotros lo hacemos hasta sexto grado como la profesora aquí.

Docente 1: Tener en claro es en lo que nos hemos propuesto ¿No?

Docente 2: Aja, ¿Cómo vas a saber si funciona o si no funciona?

Entrevistadora: Claro.

Docente 2: ¿Si hemos diversificado bien o diversificado mal?

Docente 1: Y eso hemos pedido, hemos pedido nosotros a dirección [...] cuando hemos pedido a la dirección de que esto también, la diversificación que hemos hecho, que falta reajuste y muchas cosas [...] "Que se aplique en secundaria", dijo. "Sí, sí se va hacer", pero yo no los he visto a los profesores que se han reunido, es más, que hayan visto de nosotros lo que hemos avanzado y en base a ello también quizás crear nuevas ideas.

(Grupo focal con docentes, Huaylas 02)

Es posible señalar entonces que en la escuela de Huaylas 02 existe una falta de coordinación a nivel institucional -que se observa claramente en los dos aspectos centrales de la propuesta EIB antes expuestos- que permite comprender las diferencias en los procesos pedagógicos en el aula de los dos docentes observados. Es decir, la situación de esta escuela da cuenta de cómo las dificultades a nivel de la gestión institucional y del liderazgo del director conllevan a que, en la práctica, cada docente maneje una propuesta pedagógica individual e incluso discordante con la de otros docentes.

La inclusión de conocimientos de la cultura local en las prácticas pedagógicas, también, fue un aspecto observado en las otras dos escuelas. Mientras que en la clase de 3er grado de Huaylas 02 los conocimientos de la cultura local fueron usados para el desarrollo de la clase; en Huaylas 03 y 04, este tipo de situaciones se registraron al inicio de la sesión y aisladas del desarrollo del tema de la clase. Asimismo, en estas dos escuelas, los elementos introducidos fueron huaynos, lo que supone restringir la inclusión de los conocimientos de la cultura local a elementos más bien folclóricos. A pesar de estas diferencias, cabe resaltar que un aspecto transversal a las tres escuelas es que dichos conocimientos son incluidos muy esporádicamente. 
Los comentarios de los docentes en cuanto a la diversificación curricular -que es el proceso que orienta la inclusión de conocimientos de la cultura local en los documentos de planificación educativa- nos dan cuenta de un discurso muy similar en las tres escuelas. Los docentes comentan la importancia de incluir las "costumbres, tradiciones, conocimientos y valores" locales. Los docentes de la escuela EIB (Huaylas 02) plantearon que podrían presentarse dos dificultades en el proceso de incluir dichos saberes al aula. En primer lugar, se nombró la falta de tiempo, tanto de los docentes para recoger la información de los pobladores de la comunidad, como de los padres de familia o sabios de la comunidad para hacer demostraciones de sus saberes tradicionales en la escuela. Un segundo problema es la barrera lingüística: los docentes que no dominan la lengua originaria de los pobladores tienen dificultades para recoger información en la comunidad.

Entrevistadora: [...] ¿Cómo es, cómo es el trabajo con los sabios? ¿Se trabaja? O solo digamos, se menciona, se conoce ¿Cómo se integra esto?

[Silencio]

Docente 1: Yo no lo integro.

Docente 2: En papel [...] En el diseño curricular, está.

Docente 1: Sí, está en el diseño curricular, pero no. Yo al menos, por eso, digo: "no, no lo hago extensivo". Porque deberías coger a ellos para tu proceso de enseñanza, pero por el tiempo y a veces ya cuando lo solicitas ellos te dicen tal día ¿no? Pero no puedo.

[...]

Docente 1: O para recolectar sus tradiciones, por ejemplo, ellos saben bastante de sus mitos, sus costumbres, pero, para plasmarlo pero es, es...

Docente 2: ... el problema es la comunicación.

Entrevistadora: Ya, claro.

Docente 2: Fue una... yo me quedaba más antes, trabajaba y me quedaba a dormir aquí.

Entrevistadora: Uhm.

Docente 2: Toda la semana vivía acá y ese era mi problema, pues, no salía de mi cuarto, porque cuando yo me comunicaba con ellos, ellos me hablaban un quechua fluido [...] que me hacían volar.

(Grupo focal con docentes, Huaylas 02)

A pesar de estas dificultades, se observa que la docente de 3er grado de Huaylas 02 presenta algunos conocimientos de la cultura local durante el desarrollo del tema en su clase. Empero, cabe reflexionar si dicha esporádica incorporación responde a las prácticas del Nuevo Enfoque Pedagógico (MED) o si responden a los principios de la EIB. Como lo menciona López, 
Cabe preguntarse si el hecho de que los docentes recurran ahora a las experiencias previas de los estudiantes es más bien un resultado de la apropiación de uno de los principios medulares del nuevo enfoque pedagógico y no de la asunción de uno de los sustentos del enfoque intercultural. [...] desde ambas perspectivas, se subraya la recuperación y aprovechamiento de los conocimientos previos de los educandos. Ello nos obliga a ofrecer sugerencias específicas a los maestros y maestras sobre cómo operativizar tal noción [la de interculturalidad]. (2002, p. 154)

En líneas generales, a partir de la información recogida podemos señalar que la experiencia escolar de los estudiantes indígenas en las tres escuelas observadas no responde a sus características lingüísticas ni culturales. Las situaciones observadas y reportadas por los docentes dan cuenta de un uso esporádico del quechua, centrado en facilitar la instrucción en castellano, lengua que se prioriza en las tres escuelas. Del mismo modo, los conocimientos de la cultura local son incorporados para el desarrollo de los contenidos de la clase solamente por una de los cinco docentes que observamos. Como menciona Rosales, "Los niños se acomodan a la escuela, con dificultades evidentes, pero la escuela no se adapta a sus necesidades" (2012, p. 13). La cita que presentamos a continuación muestra cómo los niños se "afanan" en ajustarse a un espacio donde se espera se comuniquen en castellano. Durante la observación de aula en Huaylas 02 , se pudo notar la siguiente interacción: mientras los niños estaban haciendo un trabajo grupal, la profesora se acercó a uno de los grupos, puesto que notó que tenía problemas para escribir una idea. Le dice a uno de los niños: "Dime qué quieres decir en quechua y yo te ayudo. Dime en quechua". El niño intenta decir su idea en castellano, pero la profesora insiste en que lo diga en quechua y le dice: "No te afanes en que querer decirlo en castellano". El niño se queda callado por unos segundos y, luego, habla en voz baja una mezcla de quechua y castellano. La profesora traduce lo dicho por el niño al castellano y se lo dicta a otro niño que toma nota en el papelógrafo: "Las plantas nos sirven para leña".

En esta misma escuela, se observó una situación que, si bien aislada, resulta bastante relevante e ilustrativa. Durante una sesión de clase, la profesora de 3er grado llevó a sus alumnos al huerto de la escuela con la intención de reconocer las plantas locales e identificar sus usos. Al oír que los niños conversaban en quechua y llamaban a las plantas por su nombre en este idioma, un grupo de alumnos de 4 to de secundaria que se encontraban trabajando en el huerto empezó a reír y burlarse de ellos. Al percatarse de esto, la profesora de 3er grado exclamó en tono burlón y de reprimenda: "Es que ellos [los de 4to de secundaria] ya no hablan en quechua". 
Más allá de lo anecdótico, lo preocupante de ambas situaciones es que tienen lugar en una escuela EIB, espacio educativo pensado para ajustarse y responder a las características y necesidades de los estudiantes indígenas. En este escenario, una situación en la que un niño indígena no se siente cómodo o confiado para expresarse en su lengua materna o en la que los estudiantes mayores se burlan de los menores por usar el quechua resulta muy preocupante. Sin embargo, cabe destacar que la demanda y necesidad de una educación pertinente a las características de los niños indígenas no debería ser un privilegio de aquellos estudiantes en las escuelas EIB.

Volvemos, entonces, a la pregunta que titula este texto: ¿educación intercultural para todos? A partir de los tres casos estudiados, encontramos que el respeto y el diálogo entre la cultura local y occidental está ausente, a pesar de que -como se señala en la Ley General de Educación 28044- el principio de interculturalidad rige para todas la escuelas del Perú. Estamos convencidas de que una educación que respete las lenguas de los estudiantes y los saberes de sus comunidades facilitaría la adaptación de estos niños a la escuela, además de que motivaría sus procesos de aprendizaje. Ello se manifiesta en una de las situaciones observadas en la escuela urbana (Huaylas 04): Un niño toma un libro del Ministerio de Educación de la caja y se lo muestra al profesor. Es un libro de cuentos en quechua. El profesor lee un párrafo del cuento mientras el niño parece emocionado por escuchar un cuento en quechua y abraza a otro niño que también está a su lado. Luego, el niño deja el libro en la caja y encuentra un cuento en castellano y lo comenta en quechua con otro niño.

\section{Sobre la participación de la comunidad en la escuela}

De acuerdo al marco normativo vigente, los miembros de la sociedad tienen el deber y el derecho de contribuir al desarrollo de la educación y participar en la definición de las políticas educativas (Ley General de Educación No. 28044 , art. 3 y 22). Detrás de este postulado destaca la concepción de que la educación no es una responsabilidad restringida a los docentes o a las instituciones educativas, sino también de la familia y de la sociedad en general. En el caso de la EIB, la participación activa de las familias y otros miembros de la localidad en la educación constituye un aspecto central, vinculado a la incorporación de saberes locales así como a la democratización de la educación.

A pesar de ello, los casos analizados nos permitieron constatar que llevar estas normas y recomendaciones a la práctica no es tarea fácil. Así, en ninguna de las tres escuelas estudiadas se registró un involucramiento de 
miembros de la comunidad ajenos a las familias (autoridades, sabios, líderes, especialistas, etc.). Por otro lado, aunque la escuela promueve actividades que están pensadas para el involucramiento de los padres en el aprendizaje de los niños, como veremos más adelante, estos espacios no están alineados con las capacidades de los padres ni con sus responsabilidades laborales. Por otro lado, existen otras actividades a las que los padres son convocados con el fin de que brinden aportes económicos o de fuerza de trabajo.

Las actividades de participación diseñadas para el involucramiento en el aprendizaje de los niños consisten en las reuniones de padres de familia con los docentes y el seguimiento pedagógico en el hogar. Según lo sostenido por los docentes entrevistados, las reuniones con los padres de familia se convocan por lo menos una vez por bimestre, lo que haría un promedio de cuatro reuniones por año escolar. Por lo general, asisten más madres que padres, $y$, dado que estas son mayoritariamente quechuahablantes, en las tres escuelas las reuniones se llevan a cabo en quechua o en quechua y castellano. En tanto la mayor parte de familias realiza actividades vinculadas a la agricultura y/o ganadería, el calendario agropecuario marca la pauta de su disponibilidad de tiempo para asistir a estas reuniones.

Entrevistadora: ¿Mamita, y usted, este...va a la escuela de Marcela? Madre de Marcela: Awmi. Tiempu kaptin, mana tiempo kaptin mana aywatsu. [...] Kay tiempo, ari, más tiempo kan... kay trigu muri, ari, murikunapaq tiumpu kanllatsu... // Sí. Cuando hay tiempo acudo, pero cuando no alcanza el tiempo no voy [...] Este tiempo, pues, más tiempo tenemos... cuando es tiempo de siembra de trigo, pues, para nuestra siembra, no hay tiempo...

(Entrevista a madre de familia, Huaylas 03)

Madre de Miguel: Hay veces no más voy colegio, casi no voy a su colegio. Entrevistadora: ¿Casi no van?

Madre de Miguel: No.

Entrevistadora: ¿'Por qué así?

Madre de Miguel: Con mis animales no puedo pues, les tengo que pastear.

(Entrevista a madre de familia, Huaylas 04)

En las tres escuelas estudiadas, se registró el uso de multas como sanción ante la inasistencia de los padres a las reuniones escolares. Por ejemplo, en Huaylas 02 , este monto asciende a S/.12 por reunión, lo que puede representar una cantidad significativa para el presupuesto de ciertas familias. 
A este respecto, algunos docentes consideran que los padres acuden a las reuniones por presión económica y no porque realmente exista un interés de su parte. Sin embargo, también, sostienen que no recurrir a esa medida, los padres estarían alejados de la escuela: "Eso [su asistencia a las reuniones] viene no porque realmente haya ese interés o por tomar conciencia frente a responsabilidad que tienen como padres sino es, mayormente por temor a la multa [...] o sea son factores que condicionan pues para que el padre asista" (Grupo focal con docentes, Huaylas 02).

Como señalábamos al inicio, a pesar de que las reuniones son espacios para el involucramiento de los padres en el aprendizaje de sus hijos, los docentes manifestaron que las intervenciones de las madres de familia durante las reuniones son mínimas o inexistentes, y que cuando intervienen, mayormente, es para indagar por las contribuciones económicas a la escuela (cuotas y actividades profondos) y no por el rendimiento de sus hijos. Las madres entrevistadas corroboraron estas afirmaciones y, como se ilustra a continuación, algunas de ellas sostuvieron que no se atreven a participar durante las reuniones debido a que se sienten avergonzadas.

Entrevistadora: ¿Mamita, y este... kaptin chay reuniónkuna, imakunata parlayanki chay reuniónkunapi? // ¿Mamita, y este... cuando hay esas reuniones, de qué cosas hablan en aquellas reuniones?

Madre de Marcela: ¿Noqa, mamita? // ¿Yo, mamita?

Entrevistadora: Ajá.

Madre de Marcela: Mana, ni imapis noqa parlapatsu, penqakumi parlapapti. // No, yo no hablo nada, me avergüenzo cuando hablo.

Entrevistadora: ¿Penqakunki? // ¿Te avergüenzas?

Madre de Marcela: Awmi. Wakinkuna parlakuyan, noqa parlakutsu. // Sí. Los demás sí participan, pero yo no hablo.

(Entrevista a madre de familia, Huaylas 03)

La poca intervención de las madres durante las reuniones y el hecho de que no indaguen por los aprendizajes de sus niños pueden deberse a barreras lingüísticas y/o de género, pero también a la verticalidad con la que se erige la relación entre docentes y madres de familia, sobre todo en los entornos rurales. En este sentido, vemos que, según a las encuestas aplicadas a los docentes, la asistencia voluntaria de los padres para informarse sobre el rendimiento de sus hijos sería mayor en la escuela urbana (Huaylas 04) que en las dos escuelas rurales. Frente a ello, es necesario tomar en cuenta que, al matricular a sus niños en la escuela, los padres hacen un "voto de confianza" en los docentes, pues los perciben como "los que saben". De esta manera, 
muchos padres esperan que cumplan su labor pedagógica al saberse poco capacitados y desprovistos de poder en este ámbito.

Entrevistadora: Y manachu aywankiku parlakunaykipaq profesoranwan, Jaime profesoranwan. // ¿Y no vas para conversar con la profesora...con la profesora de Jaime?

Madre de Jaime: Mana. Kayiratsillay, ni, kayno lichita puatsik aywapti, mamita, mana aparte aywachu. // No. "Hazle comprender", le digo, cuando así voy a hacer hervir la leche mamita, después aparte no voy.

(Entrevista a madre de familia, Huaylas 03)

Los docentes esperan que los padres no solo indaguen por el rendimiento de sus hijos en la escuela en las reuniones, sino que también brinden seguimiento pedagógico, es decir, que apoyen a los niños en casa con las tareas escolares, supervisen sus avances y absuelvan sus dudas. Según las encuestas realizadas, los docentes de ambas escuelas rurales se mostraron en desacuerdo o muy en desacuerdo ante la afirmación "los padres de mis estudiantes apoyan a sus hijos para que tengan éxito en la escuela". En Huaylas 04, por su parte, no se registró un consenso entre los docentes, aunque la mayor parte de ellos consideraba que los padres no apoyaban a sus hijos.

Complementariamente, es interesante observar la importancia que asignan los docentes de estas escuelas a la falta de apoyo de los padres a los niños. A este respecto, se observó una gradiente: en la escuela urbana (Huaylas 04), esta situación fue considerada un problema serio; en Huaylas 03, un problema moderado; y, en la escuela EIB (Huaylas 02), lo consideraron como un problema menor. Sería interesante explorar las percepciones que los docentes tienen de los padres de familia para comprender mejor el rol que se les asigna en los diferentes contextos educativos.

Los docentes encuestados de las tres escuelas coincidieron en que los PP.FF. pocas veces o nunca revisan los cuadernos y libros, o las prácticas y exámenes de sus hijos. Así, cuando los padres fallan en cumplir este rol, los docentes los califican de "indiferentes", "irresponsables", "despreocupados", etc. Sin embargo, en sus juicios y calificativos, pocos consideran que tanto los padres como las madres tienen un nivel educativo muy bajo e incluso son analfabetos funcionales ${ }^{10}$. Esta "indiferencia" o "desinterés" de los padres muchas veces se debe a que no se encuentran en capacidad de apoyar

10. Algunas madres, a pesar de haber cursado algunos años de primaria mencionaron que no sabían leer y escribir. 
o supervisar la realización de las labores escolares de sus niños en casa por falta de conocimiento. A este respecto, observamos que, salvo tres casos excepcionales ${ }^{11}$, los padres y madres tienen como máximo nivel educativo 6 to de primaria, y en promedio tienen cuatro años de escolaridad.

Entrevistadora: ¿Mamita y este... usted va a la escuela de su hijo?

Madre de Juan: Ari, aywany señorita, chaymy profesora yachanshu wamrayky manam nimuwanry // Pues, sí voy señorita; por eso, la profesora me dice que mi hijo no sabe.

Entrevistadora: Nishunky // ¿Le dice?

Madre de Juan: Chaynam ñuqaña ya preocupakuny mana lein, ymaynama kayna explikeyta puedenshu // Así pues, yo me preocupo, porque no lee; así pues, no puedo explicarle.

Entrevistadora: ¿Qam, mana yachankichu? // ¿Usted no sabe?

Madre de Juan: Aja, mana yashatshu leyta, mana kayanshu chayna, kayna mana yachayanshu, ñuqa mana kayny // Aja, no sé leer. No puedo así; como yo no sé, no puedo.

(Entrevista a madre de familia, Huaylas 02)

Además del nivel educativo de los padres, el seguimiento pedagógico que brindan a sus hijos está limitado por el poco tiempo disponible que tienen dadas sus actividades laborales vinculadas a actividades agropecuarias o al trabajo en las zonas urbanas aledañas. Si bien los estudiantes manifestaron en las encuestas y entrevistas que sus fuentes de soporte en el contexto del hogar son sus padres y hermanos, la tendencia general es considerar a estos últimos como soporte principal, sobre todo, en las escuelas rurales. De esta forma, se observó que los niños recurren a sus hermanos mayores para que les expliquen algo que no entienden o les ayuden a resolver alguna tarea. En cierto sentido, tanto las madres como los niños dejaron entrever en sus discursos que el brindar apoyo a los hermanos menores en cuestiones académicas es una de las funciones que los hermanos mayores deben asumir como parte de su rol.

Entrevistadora: Ah, ya también. Este....y acá en tu familia, en tu casa, ¿quién te puede ayudar?

Marcela: Mi hermana.

Entrevistadora: Ah, ya. Y ¿ cómo te ayuda ella? Por ejemplo, si tú no sabes hacer un problema de matemática ella ¿cómo te ayuda?

Marcela: "Así haces", me dice; y "sí, está bien", me dice el profesor.

11. En Huaylas 04, una madre alcanzó el 2do de secundaria, mientras que un padre completó estudios secundarios. En Huaylas 02, un padre cursó estudios técnicos. 
Entrevistadora: Pero ¿ella te explica o ella lo hace?

Marcela: Me explica nomás.

Entrevistadora: ¿No lo hace por ti?

Marcela: No.

(Entrevista a estudiante, Huaylas 03)

Las otras actividades escolares a las que se invita participar a los padres de familia tienen relación con sus aportes económicos y de fuerza de trabajo. En ciertos casos, los padres organizan actividades para obtener fondos para diversos fines: la reparación de la escuela, la fiesta de fin de año de sus hijos o la compra de algún material o equipo, entre otros. Asimismo, para ciertas celebraciones o actividades contribuyen con cuotas en dinero o especies, tal como se pudo observar en Huaylas 02 , cuando un niño entregó una bolsa de papas a la profesora a cuenta de su cuota del mes.

\footnotetext{
Entrevistadora: ¿Y en las reuniones qué cosa tocan?

Madre de Miguel: En el colegio, a veces, nos dicen para preparar para, celebran para Virgencita, esas cosas. "No puedo", dicen. "Un sol nomás, dos soles", así nos dicen, así pagando estoy a la profesora.

[...]

Entrevistadora: ¿Celebran a la Virgen?

Madre de Miguel: Sí. Para que preparen comida pe. Porque ese día los niños tienen que comer. Por eso nos dice a veces "es la cuota o el cuy" nos dice. Pero es de la madres acordando cuy o plata, qué cosas comprar se han de acordando.

Entrevistadora: ¿Ese cuy es para que coman los niños?

Madre de Miguel: Sí, los niños, y las profesoras.
}

(Entrevista a madre de familia, Huaylas 04)

Además, la escuela exige cuotas económicas que los padres deben abonar mensualmente para fotocopias y otros materiales educativos. Algunas de las madres entrevistadas manifestaron su malestar respecto al cobro de cuotas que consideran excesivas, y mencionaron que muchas veces exigen la rendición de cuentas de estos fondos durante las reuniones. En ciertos casos, se identificó que los niños son víctimas de amenazas, maltratos y castigos físicos por parte de los docentes por no cumplir con el pago de estas contribuciones.

"Hay veces" me dice "cuando no llevó plata"...hay veces me dijo: "mami, mami me va pegar, me va a molestar". Ayer también me dijo: "mami dice 
que no voy llevar un sol para tinta", me dijo. Por eso, yo le he molestado: “¿para qué vas a llevar plata?". Porque nosotros le hemos dado cada uno veinte soles y para qué va a necesitar plata, y cuando le he dicho, "me va jalar, me va jalar" diciendo así llorar acá. Le he dicho: "cuando te molesta vienes y yo voy a ir", le dije.

(Madre de Horacio, Huaylas 02)

Por otra parte, la participación en faenas de refacción, mantenimiento y construcción de la escuela es otra de las formas en las que los padres de familia colaboran con la escuela. En cuanto a la participación de los padres de familia en el entorno escolar, se ha identificado cierta división por género. Según algunos entrevistados, por lo general, son los padres quienes participan en las faenas y las madres quienes asisten a las reuniones. Los padres se organizan en grupos de trabajo para las faenas y su inasistencia conlleva al pago de una multa, que por ejemplo en Huaylas 02 asciende a S/. 20.

Entrevistadora: Y Chay trabajokuna mejoranku... qamkunachu rinkichiq... ustedes van a trabajar... // Y esos trabajos...mejora... ¿ustedes van a trabajar?

Madre de Jaime: Achay, mamita // Sí, mamita.

Entrevistadora: ¿... en la escuela?

Madre de Jaime: Awmi, mamita. Espusuymi aywan trabajakmi. // Sí mamita, mi esposo va a trabajar.

Entrevistadora: ¿Ahí él trabaja?

Madre de Jaime: Awmi. // Sí.

Entrevistadora: Imatakunata ruwanku? // ¿Qué cosas hace?

Madre de Jaime: Tsaynu pisunkunata, shumaq mejorayan, pintayan... Ari, tsay jaridinkunata hina mejorayan. Tseykunaman trabajayashka, mamita. // Los pisos bonito lo mejoran, pintan... Sí, los jardines también lo mejoran. En esas cosas trabajan mamita.

(Entrevista a madre de familia, Huaylas 03)

Finalmente, encontramos que en los programas de asistencia alimentaria disponibles en las escuelas la participación de las madres resulta crucial. Observamos que las tres escuelas estudiadas cuentan con el Programa de Desayunos Escolares mediante el cual el Programa Nacional de Asistencia Alimentaria (Pronaa) distribuye los insumos para los desayunos y las madres colaboran con su preparación y repartición en la escuela. Para estos efectos, ellas se organizan por turnos rotativos. 
Entrevistadora: Mamita, y usted... ¿ ¿vas a la escuela de Elsa? Madre de Elsa: Awmi. Visitarekami. Awmi. // Sí estoy visitando, sí. Entrevistadora: ¿Ima ruraqta? // ¿Para hacer qué? Madre de Elsa: A veces aywa, litsita tsuraqoq, niyamaptin... // A veces voy a poner a hervir la leche, cuando me dicen...

(Entrevista a madre de familia, Huaylas 03)

Debido al tipo de relaciones que se han establecido entre las escuelas y las comunidades, en ninguno de los casos analizados se logró identificar cambios promovidos desde la comunidad para la adaptación la escuela a las características de los estudiantes indígenas. Además, no se ha identificado una relación entre la escuela y actores comunales más allá de los padres de familia. En general, la participación de los padres en estas escuelas está más enfocada en cuestiones económicas y en el apoyo que ellos pueden brindar con su tiempo y trabajo a la institución, que en los aprendizajes de sus hijos. Esta situación coincide con lo reportado en el informe de la Defensoría del Pueblo sobre la EIB (2011), en el que se sostiene que las coordinaciones entre los padres de familia y la escuela están mayormente relacionadas a actividades de índole material y económica, en lugar de aspectos educativos y formativos de los niños. Ello no debería sorprendernos si tomamos en cuenta que los padres tenderán a involucrarse en aquellas cuestiones que manejan mejor, y, dado su bajo nivel educativo, el ámbito académico no constituye una de sus fortalezas.

Sin embargo, los padres de familia tienen un gran conocimiento de la lengua indígena y de la cultura local, y hacia ello debería apuntar su participación en la escuela. Lejos de centrarnos en sus carencias, se trata de identificar sus posibles aportes a la educación de sus hijos. Así pues, la participación de los padres y otros miembros de la comunidad debería girar en torno a la incorporación de los saberes locales en la escuela. Ello permitirá un involucramiento mucho más activo y efectivo de los padres de familia en el soporte académico de los estudiantes; un empoderamiento de la población local a partir de sus propios saberes, conocimientos y habilidades; y el establecimiento de una nueva relación padre-docente mucho más horizontal y democrática, en la que se valoren los conocimientos y capacidades de los padres de familia. 


\section{Reflexiones finales}

A lo largo de este artículo, hemos presentado algunas evidencias que nos permiten señalar que la experiencia escolar de los estudiantes indígenas en los tres casos investigados no responde a sus características lingüísticas y culturales, ni se promueven espacios reales para que los padres de familia colaboren en la educación de sus hijos.

A partir de los hallazgos, podemos señalar algunos elementos que podrían favorecer a la adaptación de niños indígenas al espacio escolar. Un primer aspecto es que la lengua materna de los niños ocupe un rol efectivo en el aula y no solo un rol funcional al castellano, como se observó en las escuelas estudiadas. Un segundo aspecto sería el de la consideración de los conocimientos locales, que deberían ser incorporados en el currículo más allá de los elementos folclóricos de las comunidades. En tercer lugar, resulta necesario que la escuela proponga nuevos espacios para la participación de los padres de familia y de otros miembros de la comunidad, pero sobre todo que reflexione sobre sus formas de relacionarse con los padres y las madres.

En suma, la puesta en marcha de estos tres aspectos supone pensar cambios coordinados entre docentes, y de los docentes con los padres de familia. Consideramos vital que los directores, profesores, padres y madres compartan las metas a las que esperan que los niños lleguen y que se discutan las mejores estrategias de enseñanza para los estudiantes. Solo así la experiencia educativa de los niños indígenas podrá ser pertinente y acorde a sus características.

Reconocemos que este es un reto amplio, sobre todo, donde las características lingüísticas y culturales de los niños y sus familias son variadas. Sin embargo, este reto no puede ser dejado de lado. Actualmente, desde el Ministerio de Educación, se vienen realizando acciones claras en pos de ello. Ejemplo de ello es el plan estratégico de EIB, el documento de la propuesta pedagógica de EIB (en consulta) y el desarrollo del primer currículo intercultural. Esperamos que estas y otras iniciativas sean incorporadas por las escuelas y permitan brindar a los estudiantes indígenas una experiencia educativa más acorde con sus necesidades y, así, desarrollar plenamente sus capacidades. 


\section{Referencias}

Defensoría del Pueblo (2011). Informe Defensorial № 152: Aportes para una Política Nacional de Educación Intercultural Bilingüe a favor de los pueblos indígenas del Perú. Lima: Defensoría del Pueblo.

Hornberger, N. (2003). La enseñanza de y en quechua en el PEEB. En I. Jung y L.E. López (Comps.), Abriendo la escuela: Lingüística aplicada a la enseñanza de lenguas (pp. 15-21). Madrid: Morata.

Indepa (2010). Mapa etnolingüístico del Perú 2010. Recuperado de: www. indepa.org.pe

Jung, I. y López, L.E. (2003). Abriendo la escuela: Perspectivas desde el lenguaje. En I. Jung y L.E. López (Comps.), Abriendo la escuela: Lingüística aplicada a la enseñanza de lenguas (pp. 15-21). Madrid: Morata.

Lopez, L. (2002). “A ver, a ver... ¿quién quiere salir a la pizarra? ¿jumasti? ¿jupasti?" Cambios iniciales en la escuela rural bilingüe peruana. Lima: MED.

Ministerio de Educación del Perú (2004). Lineamientos de política de la Educación Bilingüe Intercultural. Dirección Nacional de Educación Bilingüe Intercultural - Dinebi.

Ministerio de Educación del Perú (2012, mayo). Hacia una educación intercultural bilingüe de calidad. Propuesta pedagógica. Documento en consulta.

Rosales, E. (2012, abril). "Yo así cuando voy a otro pueblo no sé el castellano, no puedo hablar con nadie, soy muda". Usos y actitudes hacia el castellano y las lenguas originarias en la escuela pública rural. Boletín de Políticas Públicas sobre Infancia (6). Niños del Milenio.

Sanga, E. (2006). Lo que se dice y hace de la educación bilingüe intercultural en una comunidad aimara en transición en el departamento de PunoPerú (tesis de maestría no publicada).Cochabamba: Universidad Mayor de San Simón.

Tapia, Y. (2002). Estrategias metodológicas de enseñanza y uso de lenguas en docentes de escuelas EBI en el distrito de Mañazo-Puno (tesis de maestría no publicada). Cochabamba: Universidad Mayor de San Simón.

Unicef (2012, 12 de junio). Entrevista a Carmen López, Oficial de Educación de Unicef. Recuperada de: http://www.unicef.org/peru/spanish/education_3946.htm

Zavala, V. (2001). Oralidad y escritura en la educación bilingüe (a propósito de interculturalidad). Lima: MED-Plancad-GTZ-KfW. 
Zavala, V. y Córdova, G. (2003). Volver al desafío: Hacia una definición crítica de la Educación Bilingüe Intercultural en el Perú. Lima: Ministerio de Educación - Dinfocad-GTZ-Proeduca-Componente de Educación Bilingüe Intercultural.

Zavala, V., Cuenca, R. y Córdova, G. (2005). Hacia la construcción de un proceso educativo intercultural: Elementos para el debate. Lima: MED-Dinfocad-Proeduca-GTZ.

Zúñiga, M. (2008). La educación intercultural bilingüe: El caso peruano. Lima: Foro Latinoamericano de Políticas Educativas. 